\title{
$\alpha-1,3$ Glucan Synthesis Correlated with $\alpha-1,3$ Glucanase Synthesis, Conidiation and Fructification in Morphogenetic Mutants of Aspergillus nidulans
}

\author{
By B. J. M. ZONNEVELD \\ Genetisch Laboratorium, Kaiserstraat 63, Leiden, The Netherlands
}

(Received 24 September 1973; revised 26 October 1973)

\section{SUMMARY}

Nine mutants of Aspergillus nidulans differing in cleistothecia and/or conidia production were investigated. The strains were grown on four media with different levels of glucose $(0.8,3$, and $4 \%)$ and nitrate $(0.6$ and $0.15 \%)$. The mycelia were analysed on the basis of the dry weight of the total mycelium and the alkalisoluble fraction (containing the $\alpha-1,3$ glucan) as well as enzyme activities lytic to $\alpha-\mathrm{I}, 3$ glucan, laminarin, and starch. Low quantities of $\alpha-\mathrm{I}, 3$ glucan present on the third day are correlated with low $\alpha-1,3$ glucanase activity and absence of cleistothecia formation on the sixth day. $\alpha-I, 3$ Glucan and cleistothecium formation seemed to be inversely related to conidiation.

\section{INTRODUCTION}

Organisms that are amenable to both biochemical/genetic and morphogenetic investigation are the most suitable objects for the study of morphogenesis. Aspergillus nidulans has the additional advantage of having been intensively investigated genetically. An earlier study (Zonneveld, $1972 b$ ) demonstrated interdependence between the formation of $\alpha-1,3$ glucan in the cell wall, the appearance of the developmentally regulated enzyme $\alpha-1,3$ glucanase, the breakdown of the $\alpha-1,3$ glucan and cleistothecium development. In the presence of 2-deoxy-glucose, formation of both $\alpha-1,3$ glucan and cleistothecia could be specifically prevented without severely affecting growth (Zonneveld, I973). I report the results of various biochemical analyses in nine morphogenetic mutants differing with respect to production of cleistothecia and conidia.

\section{METHODS}

Organism and growth conditions. The strains of Aspergillus nidulans (Eid.) Winter were derived from the wild-type strains biAI (ATCC24704) or $y, b i A I ; y, b i A I, a c l-4 ; y, b i A r$, $a c l-5 ; y, b i A I, l c l-I 4 ; b i A I, d c l-I$; and $b i A I, a c l-I$. They were isolated by visual inspection of cleistothecial density after u.v.-irradiation. Three strains were kindly provided by $\mathrm{Dr}$ A. J. Clutterbuck (see Clutterbuck, 1969): biAr, medAr5; biAr, brlAI; and biAI, stuAI. These were isolated as affected in conidial development by visual inspection. Significance of symbols: $a c l$, acleistothecial; $l c l$, low cleistothecial; $d c l$, dense cleistothecial; med, medusa-like sterigmata; $b r l$, bristle-like conidiophores; stu, stunted conidiophores; $b i$, biotin dependent; $y$, yellow conidia. Further description of the strains is given in Results. Unless indicated, medium and growth conditions were as described earlier (Zonneveld, I $972 b$ ) except that ascospores were used for inoculation of aconidial strains.

Analytical procedures. Enzyme isolation and mycelium isolation and fractionation were 
carried out as described elsewhere (Zonneveld, 1973). Enzymes were assayed as follows: for laminarin and soluble starch $(0.55 \mathrm{mg} / \mathrm{ml})$ and $\alpha-1,3$ glucan $(2 \mathrm{mg} / \mathrm{ml}), 0.9 \mathrm{ml}$ portions were incubated with $0.2 \mathrm{ml}$ of a tenfold diluted enzyme preparation. All solutions were made with citrate-phosphate buffer $\mathrm{pH} 6 \cdot 2$.

The increase in reducing power was measured with the neocuproin reagent (Dygerts, Li, Florida \& Thoma, 1965). Enzyme activities are expressed in units per plate (containing $50 \mathrm{ml}$ of medium), one unit being the amount of enzyme that liberates I $\mu \mathrm{mol}$ of glucose $/ \mathrm{min}$ at $37^{\circ} \mathrm{C}$. Units per plate were chosen instead of per $\mathrm{mg}$ dry weight or per $\mathrm{mg}$ protein because these increase and decrease with the age of the cultures. All experiments were carried out at least three times. The figures in Table $\mathrm{I}$, however, were from one experiment. Although there was some variation between the experiments, the figures within each experiment all have the same relative values.

\section{RESULTS}

Four different media were used in the experiment for the following reasons. Medium with $3 \%$ glucose and $0.6 \% \mathrm{NaNO}_{3}$ is the standard medium and is also included for comparison with earlier results (Zonneveld, $1972 a, b$; 1973). Media with higher quantities of glucose $(4 \%)$ or lower quantities of nitrate $(0.15 \%)$ were chosen because more $\alpha-1,3$ glucan was formed and it was believed that this might result in repair of the cleistothecialdefective strains. On medium with $0.8 \%$ glucose low quantities of $\alpha-I, 3$ glucan were found and no fructification (cleistothecium formation) took place, showing the importance of $\alpha-I, 3$ glucan. Maximum dry weights were reached when glucose was exhausted from the medium. This took place with 3,4 and $0.8 \%$ glucose (all with $0.6 \% \mathrm{NaNO}_{3}$ ) and $3 \%$ glucose $\left(0.15 \% \mathrm{NaNO}_{3}\right)$ respectively on the third, fourth, second and fourth days. Maximum enzyme activities were found respectively on the sixth, sixth, third and sixth days.

\section{Development in wild type biAI}

On the standard medium a thick mycelial mat was formed $24 \mathrm{~h}$ after inoculation on $50 \mathrm{ml}$ agar plates, and conidiospores were formed during the next $48 \mathrm{~h}$. The latter were borne on two tiers of sterigmata which in turn had developed from the swollen apex (vesicle) of conidiophores. On the third day, the maximum dry weight was attained and the glucose in the medium was exhausted. Table I shows that on standard medium about one-third of the dry weight was accounted for by the alkali-soluble fraction containing the $\alpha-1,3$ glucan. For comparison, measurements were made of laminarinase and amylase: enzymes which split respectively $\beta-\mathrm{I}, 3$ and $\alpha-\mathrm{I}, 4$ linkages of glucans found in the alkali-insoluble fraction of the cell wall.

Both the absolute and the relative amounts of $\alpha-1,3$ glucan were strongly dependent on the amount of glucose in the medium (Table I). The low activity of $\alpha-\mathrm{I}, 3$ glucanase on $0.8 \%$ glucose was correlated with the presence of low quantities of $\alpha-1,3$ glucan, but the laminarinase and amylase levels were not depressed. The presence of $\alpha-1,3$ glucan was not the only obligatory factor for enzyme production, because on a low-nitrate medium all enzyme activities were low (including that of $\alpha-I, 3$ glucanase, despite the presence of a relatively large amount of $\alpha-\mathrm{I}, 3$ glucan). Cleistothecia developed abundantly (about 50000 per plate) on a medium containing 3 or $4 \%$ glucose. With $0.8 \%$ glucose very little $\alpha-I, 3$ glucan was formed, and there were no cleistothecia. In the presence of $0.15 \%$ nitrate $(3 \%$ glucose) a measurable quantity of glucan was produced but the amount of $\alpha-\mathrm{I}, 3$ glucanase was undetectable and there were few cleistothecia. 


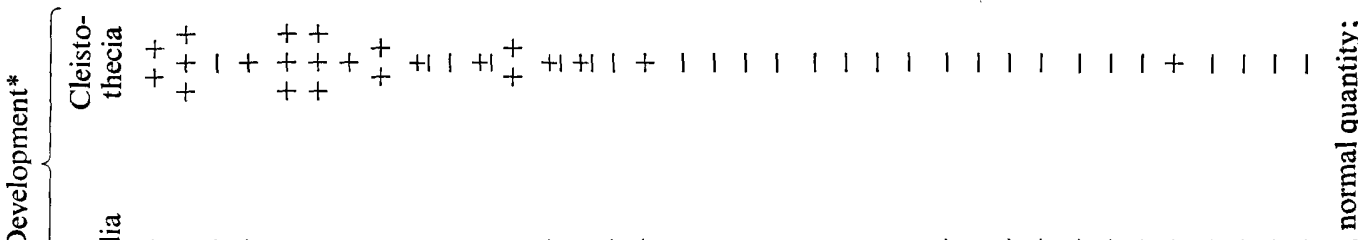

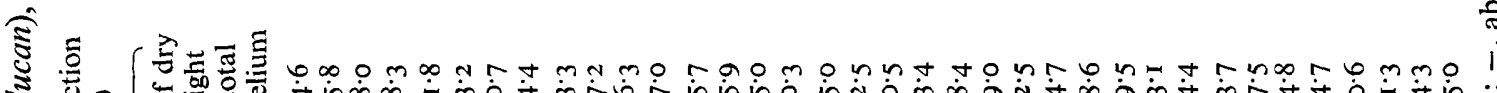

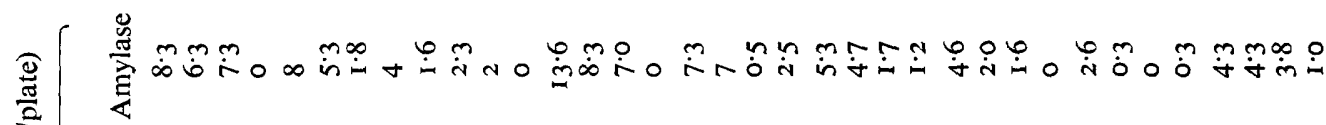

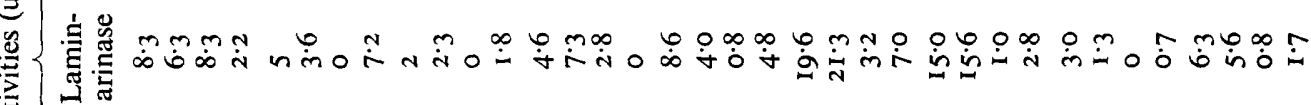
ट्ठ

空

营

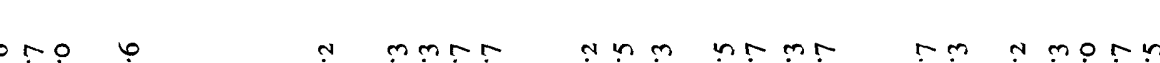

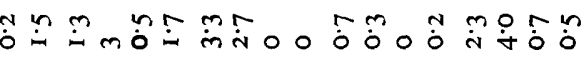




\section{Development in morphological mutants}

Strain biAI, dcl-r. After three days' growth this strain, which produces few conidia, was covered by a thick layer of Hülle cells (preceding cleistothecium development) giving it a golden-yellow appearance and a resemblance to the strain described as $d p$ by Mahoney $\&$ Wilkie (1962). On the standard medium, more than twice the amount of $\alpha$-I,3 glucan was produced as compared with the wild type. No conidia were formed on the higher quantities of glucose, possibly due to the large amount of $\alpha-\mathrm{I}, 3$ glucan synthesized.

In the wild type almost $25 \%$ of the dry weight is accounted for by conidia, as measured on the seventh day (Zonneveld, $1972 b$ ). The very large number of cleistothecia found on all media, as compared with the wild type, might be the result of the high $\alpha$-I, 3 glucan level. Enzyme formation is considerable on a low-nitrate medium as compared with the wild type. The small number of conidia on this medium might leave more nitrogen for enzyme formation.

Strain biAI, brlAI. This strain was unable to form conidia. Its conidiophores failed to make vesicles or subsequent structures and continued to grow as long bristles with 20 to 30 times the normal length (Clutterbuck, 1969; Oliver, 1972). If the strain is point-inoculated on $3 \%$ glucose, a normal number of cleistothecia develop, but when, as in these experiments, a spore suspension is spread over the agar surface, only a few cleistothecia develop on the standard medium. The whole plate was covered by a thick cotton-like layer consisting of conidiophores. As can be seen from Table I, glucan formation and enzyme production were low, again indicating a relationship with fructification. On low nitrate there were normal amounts of $\alpha-\mathrm{I}, 3$ glucan and cleistothecia. $\alpha-\mathrm{I}, 3$ Glucanase could not be detected; however, laminarinase and amylase also showed low activity on all media.

Strain $y, b i A I, l c l-14$. This yellow-spored strain formed a normal amount of conidia but few cleistothecia developed on the standard medium. It synthesized more $\alpha$-I, 3 glucanase than any of the other strains and also produced a large amount of $\alpha-I, 3$ glucan. Nevertheless, cleistothecium formation was impaired, although less on a low-nitrate medium. The high activity of $\alpha-\mathrm{I}, 3$ glucanase conceivably resulted in a high glucose level, thus suppressing fructification. Amylase activity was highest in this strain, but laminarinase was normal.

Strain biAI, medAI5. This strain characteristically formed more than two tiers of sterigmata, and it was unable to form cleistothecia (Clutterbuck, 1969). The impaired change from sterigmata to conidia formation might be related to the change from Hülle cells to the formation of cleistothecia. The same mutation probably governs both characters.

Revertants of this strain show progress in conidia formation roughly correlated with cleistothecium development (A. J. Clutterbuck, personal communication). However, $\alpha$-I, 3 glucanase production was normal and $\alpha-1,3$ glucan was even higher than in the wild type, especially in the presence of low quantities of nitrate. The $\alpha-1,3$ glucan was in fact degraded during development (unpublished result).

Besides this strain, four other acleistothecial strains having several characteristics in common were studied.

Strain biAI, stuAI. This strain formed stunted conidiophores and produced few conidia. There seemed to be a general lack of wall thickening and no cleistothecia were produced (Clutterbuck, I969).

Strain biAI, acl-r. Strain biAI, acl-I was classified in our laboratory as unable to form a heterokaryon. Testing revealed that it was also unable to produce cleistothecia.

Strains $y, b i A I$, acl-4 and $y, b i A I$, acl-5. These strains, which were induced by u.v. in the strain $y, b i A I$, were characteristically unable to produce cleistothecia. 


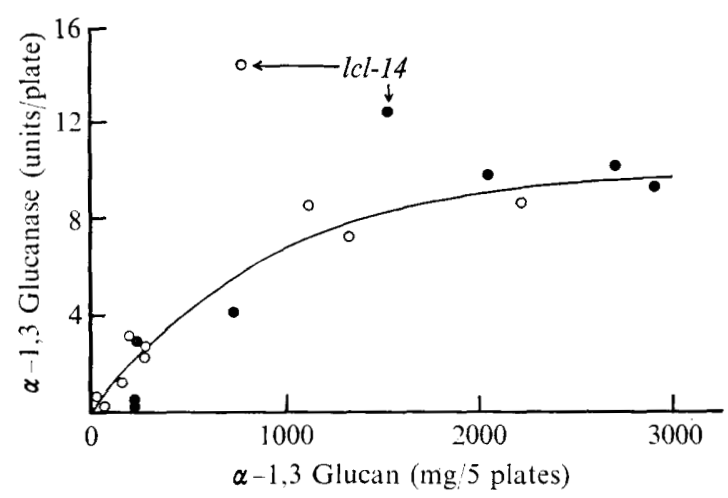

Fig. I. Correlation between the synthesis of $\alpha-1,3$ glucan and the activity of $\alpha-1,3$ glucanase on media with $3 \%(O)$ and $4 \%(\odot)$ glucose by the nine mutants of $A$. nidulans.

All four were investigated on the various media and all showed a very low quantity of $\alpha-I, 3$ glucan and $\alpha-1,3$ glucanase (Table I). This phenomenon might be specific, because the enzymes laminarinase and amylase were produced in normal amounts. The level of laminarinase activity in $s t u A I$ and $a c l-I$ was twice that of the wild type. All four strains produced the highest quantity of $\alpha-\mathrm{I}, 3$ glucan on the low-nitrate medium, and cleistothecia were formed even in strain $y, b i A I, a c l-4$ (Table I). In strains $b i A r, a c l-I$ and $b i A I$, stuAI, the addition of a suspension of $\alpha-\mathrm{I}, 3$ glucan to the medium did not result in fructification.

\section{DISCUSSION}

The biochemical data on the $\alpha$-I,3 glucan $/ \alpha-\mathrm{I}, 3$ glucanase system point to an involvement in the regulation of development in Aspergillus nidulans. If sufficient $\alpha-1,3$ glucan was formed and the glucose supply was exhausted, $A$. nidulans synthesized the enzyme $\alpha-\mathrm{I}, 3$ glucanase. This enzyme degraded $\alpha-\mathrm{I}, 3$ glucan, thus supplying the necessary carbon and energy for fructification (Zonneveld 197 I $; 1972 a, b$ ).

The following remarks can be made about the present results:

$\alpha$-I,3 glucan synthesis. $\alpha-\mathrm{I}, 3$ glucan will be synthesized under the following three conditions:

I. There must be sufficient glucose in the medium; $0.8 \%$ glucose is inadequate for the wild type.

2. Conidia or related structures (conidiophores) should not consume too much glucose. On $3 \%$ glucose, the continuously growing conidiophores of the $b r l A I$ strain consumed the amount of glucose used by the wild type for $\alpha-1,3$ glucan formation. Apparently, the conidiophores themselves do not contain significant amounts of $\alpha-1,3$ glucan. On low quantities of nitrate, a high quantity of glucan occurred in all strains, probably because conidiophore growth stopped due to lack of nitrate but glucan formation continued. The same observation was made with Aspergillus aculeatus, where nigeran accumulation was enhanced under nitrogen limitation, although this acleistothecial organism was unable to degrade the latter (Gold, Mitzel \& Segel, 1973). Although it might be assumed that under a nitrate deficiency the organism is unable to form either $\alpha-1,3$ glucanase or cleistothecia, it was found that the amount of proteinase increased under this condition (unpublished results), and this could supply the required nitrogen.

3. The genetic factors involved in $\alpha-1,3$ glucan synthesis must function properly. (In stuAI, acl-4, acl-5, and acl-I, they apparently do not.) 
$\alpha$-I,3 glucanase synthesis. The degrading enzyme $\alpha$-I,3 glucanase will be formed under the following conditions:

I. The glucose in the medium must be exhausted, since it is known that this enzyme is under glucose repression (Zonneveld, I972 $b$ ).

2. The amount of $\alpha-1,3$ glucan must be adequate, because this glucan regulates the level of the $\alpha-I, 3$ glucanase in some way. If low quantities of $\alpha-I, 3$ glucan were synthesized, either experimentally or due to a genetic defect, this has so far always been found to be correlated with a low activity of $\alpha-1,3$ glucanase. For example, $0 \cdot 1 \% 2$-deoxy-glucose added to a $3 \%$ glucose medium or plain $0.8 \%$ glucose gave very low quantities of $\alpha-\mathrm{I}, 3$ glucan; subsequently, very low quantities of enzyme were formed (see above, and Zonneveld, I973). The same was found in four acleistothecial strains synthesizing low quantities of glucan. With wild type, $d c l-r$ and medAI5, increased glucan production at various glucose concentrations was correlated with an increase in the $\alpha-I, 3$ glucanase concentration up to a maximum level (Fig. I).

3. Enough nitrate must be available. The organism began by forming mycelium and $24 \mathrm{~h}$ later conidia formation and $\alpha-\mathrm{I}, 3$ glucan synthesis increased sharply. Conidia formation decreased as soon as the nitrate was exhausted, but the synthesis of $\alpha-1,3$ glucan continued with the available glucose. Under this condition the synthesis of $\alpha-\mathrm{I}, 3$ glucanase was dependent on the degradation of nitrogen-containing cellular material. Therefore, enzyme activity was low and only a small number of cleistothecia were formed.

4. A strain producing $\alpha-\mathrm{I}, 3$ glucan but not $\alpha-\mathrm{I}, 3$ glucanase has not yet been isolated, but such mutants can be expected because Riedel, Gerisch, Müller \& Beug (I973) found morphological mutants in Dictyostelium discoideum that were defective in the cAMPcAMP-phosphodiesterase system with respect to cAMP phosphodiesterase.

Cleistothecium and conidium formation. If $\alpha-\mathrm{I}, 3$ glucan and $\alpha-\mathrm{I}, 3$ glucanase are synthesized in adequate amounts, cleistothecia can be formed except when a mutation is expressed, presumably at a later stage (lcl-14, medAI5). The more $\alpha-1,3$ glucan that was available, the more cleistothecia were formed. This was clearly demonstrated in strain $d c l-I$. In strain acl-4 cleistothecia were formed on the low-nitrate medium. Apparently, this strain only becomes deficient in this respect if the glucan content is below a certain level. In the other three strains the absence of cleistothecium formation despite the glucan available on lownitrate medium, might be explained by expression of the mutation at a later stage of development. So far, the results of experiments as well as the properties of mutants point to a special role in cleistothecium development of $\alpha-I, 3$ glucanase in combination with $\alpha-I, 3$ glucan. The activities of laminarinase and amylase seemed to vary independently. The data suggest that, as compared to the other glucanases in the organism, the specific role of $\alpha-1,3$ glucanase lies in the fact that the level is regulated by the amount of $\alpha-1,3$ glucan and that it might form the starting point for the developmental programme leading to cleistothecium formation.

Fig. I shows that the amounts of $\alpha-\mathrm{I}, 3$ glucan and $\alpha-\mathrm{I}, 3$ glucanase were correlated although the values were derived from nine different strains on media containing 3 and $4 \%$ glucose (Table I). It seemed that above a certain level of glucan, the amount of $\alpha-\mathrm{I}, 3$ glucanase ceased to increase. Mutant $l c l-14$ was clearly distinguished by its absolutely and relatively high quantity of $\alpha-\mathrm{I}, 3$ glucanase. The number of conidia seemed roughly inversely proportional to the number of cleistothecia, and decreasing amounts of conidia were found with increasing amounts of glucose in the medium. Higher quantities of glucose lead to the production of more $\alpha-\mathrm{I}, 3$ glucan. This effect was reinforced by the reduced formation of conidia, which meant that a greater proportion of the glucose was available for $\alpha-I, 3$ glucan 
synthesis. It is conceivable that the rapid synthesis of large amounts of $\alpha-\mathrm{I}, 3$ glucan prevented conidiophore initials from breaking through the cell wall, which thickened at an early stage. For instance, mutant $d c l-I$ made large amounts of glucan and conidia formation was suppressed.

These conclusions are in part speculative and should be regarded mainly as offering a basis for further experiments on the regulation of morphogenesis and the function of protein degradation in $A$. nidulans.

\section{REFERENCES}

Clutterbuck, A. J. (1969). A mutational analysis of conidial development in Aspergillus nidulans. Genetics 63, 317-327.

Dygerts, S., Li, L. H., Florida, D. \& Thoma, J. A. (1965). Determination of reducing sugar with improved precision. Analytical Biochemistry I3, 367-374.

Gold, M. H., Mrtzel, D. L. \& Segel, I. H. (1973). Regulation of nigeran accumulation by Aspergillus aculeatus. Journal of Bacteriology II3, 856-862.

MAHONEY, M. \& WiLKIE, D. (1962). Nucleo-cytoplasmic control of perithecial formation in Aspergillus nidulans. Proceedings of the Royal Society B I56, 524-532.

Oliver, P. T. P. (1972). Conidiophore and spore development in Aspergillus nidulans. Journal of General Microbiology 73, 45-54.

Riedel, V., Gerisch, G., Müller, E. \& Beug, H. (I973). Defective cyclic adenosine-3', $5^{\prime}$ phosphatephosphodiesterase regulation in morphogenetic mutants of Dictyostelium discoideum. Journal of Molecular Biology 74, 573-585.

ZoNNEveLD, B. J. M. (197I). Biochemical analysis of the cell wall of Aspergillus nidulans. Biochimica et biophysica acta 249, 506-5I I.

ZONNEVELD, B. J. M. (1972a). A new type of enzyme, an exo-splitting $\alpha-1,3$ glucanase from non-induced cultures of Aspergillus nidulans. Biochimica et biophysica acta 258,54I-547.

ZONNEVELD, B. J. M. (1972b). Morphogenesis in Aspergillus nidulans: the significance of $\alpha-\mathrm{I}, 3$ glucan of the cell wall and $\alpha-1,3$ glucanase for cleistothecium development. Biochimica et biophysica acta 273, 174-187.

ZONNEVELD, B. J. M. (1973). Inhibitory effect of 2-deoxy-glucose on cell wall $\alpha-\mathrm{I}, 3$ glucan synthesis and cleistothecium development in Aspergillus nidulans. Developmental Biology 34, $\mathrm{I}-8$. 